\title{
Phase Relations Between Iridium and the Sesquioxides in Air
}

\author{
S. J. Schneider, J. L. Waring, and R. E. Tressler ${ }^{1}$
}

(February 11, 1965)

\begin{abstract}
A study has been made by x-ray diffraction analysis of the reactions that occur in an air environment between $\mathrm{Ir}$ and $\mathrm{IrO}_{2}$ and each of the following: $\mathrm{Nd}_{2} \mathrm{O}_{3}, \mathrm{Sm}_{2} \mathrm{O}_{3}, \mathrm{Eu}_{2} \mathrm{O}_{3}, \mathrm{Gd}_{2} \mathrm{O}_{3}, \mathrm{Dy}_{2} \mathrm{O}_{3}, \mathrm{Ho}_{2} \mathrm{O}_{3}, \mathrm{Y}_{2} \mathrm{O}_{3}$, $\mathrm{Er}_{2} \mathrm{O}_{3}, \mathrm{Tm}_{2} \mathrm{O}_{3}, \mathrm{Yb}_{2} \mathrm{O}_{3}, \mathrm{Lu}_{2} \mathrm{O}_{3}, \mathrm{In}_{2} \mathrm{O}_{3}, \mathrm{Sc}_{2} \mathrm{O}_{3}$, and $\mathrm{Al}_{2} \mathrm{O}_{3}$. In air $\mathrm{Ir}$ oxidizes at low temperatures to form $\mathrm{IrO}_{2}$ which in turn dissociates at $1020{ }^{\circ} \mathrm{C}$. The pseudo binary $\mathrm{Nd}_{2} \mathrm{O}_{3}-\mathrm{IrO}_{2}$ was studied in detail inasmuch as it typified many of the $\mathrm{Ln}_{2} \mathrm{O}_{3}-\mathrm{IrO}_{2}$ systems. Two compounds, $\mathrm{Nd}_{2} \mathrm{O}_{3} \cdot 2 \mathrm{IrO}_{2}$ and $3 \mathrm{Nd}_{2} \mathrm{O}_{3} \cdot 2 \mathrm{IrO}_{2}$ occur in the system. The former, a cubic pyrochlore type phase, dissociates upon heating at $1190{ }^{\circ} \mathrm{C}$. The $3: 2$ compound dissociates to the solid phases, $\mathrm{Nd}_{2} \mathrm{O}_{3}$ and $\mathrm{Ir}$, at $1300{ }^{\circ} \mathrm{C}$. Prior to dissociation, the $3: 2$ compound undergoes an apparent polymorphic transition at $1195{ }^{\circ} \mathrm{C}$ which may be related to an oxygen loss. Up to at least $2000^{\circ} \mathrm{C}$ no further reaction occurred between $\mathrm{Ir}$ and $\mathrm{Nd}_{2} \mathrm{O}_{3}$. All Band $\mathrm{C}$-type rare earth oxides formed cubic pyochlore type compounds with $\mathrm{IrO}_{2}$. Each of these compounds subsequently dissociated upon heating. No apparent reaction occurred between $\operatorname{IrO}_{2}$ and either $\mathrm{In}_{2} \mathrm{O}_{3}, \mathrm{Sc}_{2} \mathrm{O}_{3}$, or $\mathrm{Al}_{2} \mathrm{O}_{3}$.
\end{abstract}

\section{Introduction}

As part of the program at the National Bureau of Standards to obtain accurate melting points of the metal oxides $[1]^{2}$, a study has been initiated to determine what effect, if any, various refractory metal containers have upon possible standard oxides. The type of reaction between appropriate container materials such as iridium (Ir) or tungsten (W) and an oxide can be best characterized through a thorough study of their equilibrium phase relations. As the first in a series of phase equilibria investigations, the present paper reports results obtained in a study of portions of the Nd-Ir-Oxygen and related systems in an air environment.

Preliminary information indicated that powdered Ir has a tendency to oxidize to $\mathrm{IrO}_{2}$ when heated in air at moderate temperatures. Since complete oxidation is difficult to achieve, $\mathrm{IrO}_{2}$ rather than Ir metal was selected as one end member of the system. By utilizing $\mathrm{IrO}_{2}$, an approach to equilibrium could be achieved more readily. The study would still reflect, however, the behavior in air of Ir metal in combination with $\mathrm{Nd}_{2} \mathrm{O}_{3}$ and other oxides.

Iridium has a face-centered cubic, copper type structure with $a=3.8394 \AA$ [2]. The freezing point

\footnotetext{
${ }^{1}$ Mr. Tressler is currently a graduate student at Pennsylvania State University.
}

${ }^{2}$ Figures in brackets indicate the literature references at the end of this paper. of Ir is $2443{ }^{\circ} \mathrm{C}$, a value which is given as a secondary reference point on the International Practical Temperature Scale $(\mathrm{IPTS})^{3}$ [3]. Iridium dioxide $\left(\mathrm{IrO}_{2}\right)$ is similar to $\mathrm{TiO}_{2}$ in having the tetragonal, rutile structure with $a=4.4983 \AA$ and $c=3.1544 \AA$ [4]. Upon heating, $\mathrm{IrO}_{2}$ has been reported to dissociate to the metal and a vapor phase at $1100{ }^{\circ} \mathrm{C}$ in one atmosphere oxygen [5].

Neodymium sesquioxide $\left(\mathrm{Nd}_{2} \mathrm{O}_{3}\right)$ has been reported to occur in three polymorphic forms; the $\mathrm{A}, \mathrm{B}$, and $\mathrm{C}$ rare earth oxide structure types $[6,7]$. The hexagonal A type ( $a=3.831 \AA, c=5.999 \AA)$ [8], is generally regarded as the stable modification of $\mathrm{Nd}_{2} \mathrm{O}_{3}$ although there has been some controversy regarding the existence and stability of the $B$ and $C$ types $[6,7]$. The melting point of $\mathrm{Nd}_{2} \mathrm{O}_{3}$ is not well established. The one value reported in the literature lists the melting point as $2272^{\circ} \mathrm{C}$ for material having a purity of 99 percent [9].

Since little data are available on either $\mathrm{Ir}$ of $\mathrm{IrO}_{2}$ in combination with oxides, the present study was broadened somewhat to include, in addition to $\mathrm{Nd}_{2} \mathrm{O}_{3}$, the following materials: $\mathrm{Sm}_{2} \mathrm{O}_{3}, \mathrm{Eu}_{2} \mathrm{O}_{3}, \mathrm{Gd}_{2} \mathrm{O}_{3}, \mathrm{Dy}_{2} \mathrm{O}_{3}$, $\mathrm{Ho}_{2} \mathrm{O}_{3}, \mathrm{Y}_{2} \mathrm{O}_{3}, \mathrm{Er}_{2} \mathrm{O}_{3}, \mathrm{Tm}_{2} \mathrm{O}_{3}, \mathrm{Yb}_{2} \mathrm{O}_{3}, \mathrm{Lu}_{2} \mathrm{O}_{3}, \mathrm{In}_{2} \mathrm{O}_{3}$, $\mathrm{Sc}_{2} \mathrm{O}_{3}$, and $\mathrm{Al}_{2} \mathrm{O}_{3}$.

\footnotetext{
${ }^{3}$ This scale (IPTS) applies to all temperatures listed in this paper.
} 


\section{Materials}

All starting materials employed in this study had a purity of 99.7 percent or greater. With the exception of $\mathrm{Ir}, \mathrm{IrO}_{2}$, and $\mathrm{Al}_{2} \mathrm{O}_{3}$, the oxides were used in previous investigations and their spectrochemical analyses are reported elsewhere $[10,11,12]$. The $\mathrm{Ir}, \mathrm{IrO}_{2}$, and $\mathrm{Al}_{2} \mathrm{O}_{3}$ samples were found by general qualitative spectrochemical analysis ${ }^{4}$ to have the following impurities:

Ir $0.01-0.1 \%$ each $\mathrm{Pd}, \mathrm{Pt}$ and $\mathrm{Si}$; 0.001-0.01\% each of $\mathrm{Ag}, \mathrm{Cu}$, and $\mathrm{Fe}$; $0.0001-0.001 \%$ each $\mathrm{Al}$ and $\mathrm{Mg}$; and $<0.0001 \%$, Ca.

$\mathrm{IrO}_{2} 0.01-0.1 \%, \mathrm{Pt}$; 0.001-0.01\% each $\mathrm{Al}, \mathrm{Cu}, \mathrm{Fe}, \mathrm{Pd}$, and $\mathrm{Si}$; $0.0001-0.001 \% \mathrm{Mg}$; and $<0.0001 \% \mathrm{Ca}$.

$\mathrm{Al}_{2} \mathrm{O}_{3}$ 0.01-0.1\% each $\mathrm{Ga}, \mathrm{Pb}$ and $\mathrm{Si}$; $0.001-0.01 \%$ each $\mathrm{Cu}, \mathrm{Fe}$, and $\mathrm{Mg}$; 0.0001-0.001\% each Ag, Ca, and Cr.

\section{Experimental Procedure}

Specimens for the oxide- $\mathrm{IrO}_{2}$ studies were prepared from $0.5 \mathrm{~g}$ batches of various combinations of the end members. Calculated amounts of each oxide, corrected for ignition loss, were weighed to the nearest milligram. Each batch was thoroughly hand mixed, placed in Pt tubes and fired at 800 or $1000{ }^{\circ} \mathrm{C}$ a minimum of $19 \mathrm{hr}$ in a muffle furnace.

Following the preliminary heat treatment, a portion of each calcined batch was placed in a small Vycor tube (sealed at one end) and fired in a $\mathrm{Pt}$ alloy quench furnace at various temperatures for different periods of time until equilibrium was attained. The Vycor tube containing the specimen was air quenched by quickly pulling the tube from the furnace. Equilibrium was assumed to have been achieved when the $\mathrm{x}$-ray pattern showed no change after successive heat treatments of a specimen or when the data were consistent with the results from a previous set of experiments.

The use of Vycor tubes instead of $\mathrm{Pt}$ for experiments above $1000{ }^{\circ} \mathrm{C}$ was necessitated by the fact that Ir, frequently found as a decomposition product, readily reacts with $\mathrm{Pt}$. On the other hand, the Vycor tube did not appear to influence or react with the various oxide samples.

Temperatures in the quench furnace, controlled to within $\pm 3{ }^{\circ} \mathrm{C}$, were measured with a 100 percent $\mathrm{Pt}$ versus 90 percent $\mathrm{Pt}-10$ percent $\mathrm{Rh}$ thermocouple. All reported temperatures pertaining to quench furnace data are considered accurate to within $\pm 5^{\circ} \mathrm{C}$.

\footnotetext{
${ }^{4}$ The spectrochemical analyses were performed by the Spectrochemical Analysis Sectio of the National Bureau of Standards.
}

Several experiments were conducted with mixtures having 1:2 mole ratios of oxide to Ir metal. Specimens of this type were prepared from two gram batches. After each batch was mechanically mixed it was heat treated at three different temperatures; specifically, $1000{ }^{\circ} \mathrm{C}$ for $12 \mathrm{hr}, 1400{ }^{\circ} \mathrm{C}$ for $1 \mathrm{hr}$ and $\approx 2000{ }^{\circ} \mathrm{C}$ for $1 / 2 \mathrm{hr}$. A small iridium pellet was used as a setter material. A program controlled tube furnace was employed for the 1000 and $1400{ }^{\circ} \mathrm{C}$ treatments. An induction furnace [11] utilizing an iridium crucible as both the susceptor and specimen container was used for the $2000{ }^{\circ} \mathrm{C}$ treatment. Temperatures were measured with a calibrated optical pyrometer sighted through a small hole in the crucible cover. The optical pyrometer temperature measurements are estimated to be accurate to within $\pm 25^{\circ} \mathrm{C}$ or better.

All specimens were examined by x-ray diffraction techniques at room temperature using a high angle recording Geiger counter diffractometer and Nifiltered $\mathrm{Cu}$ radiation.

\section{Results and Discussion}

\section{1. $\mathrm{Nd}_{2} \mathrm{O}_{3}-\mathrm{IrO}_{2}$ and $\mathrm{Nd}_{2} \mathrm{O}_{3}-\mathrm{Ir}$ Systems in Air}

The equilibrium phase diagram for the combined $\mathrm{Nd}_{2} \mathrm{O}_{3}-\mathrm{IrO}_{2}$ and $\mathrm{Nd}_{2} \mathrm{O}_{3}-\mathrm{Ir}$ systems in air is given in figure 1. The diagram was constructed from the data listed in table 1. The solid circles indicate the compositions and temperatures of the experiments conducted. It should be emphasized that figure 1 does not represent a true binary system, but a composite of the true binary, $\mathrm{Nd}_{2} \mathrm{O}_{3}-\mathrm{Ir}$, and the pseudo binary, $\mathrm{Nd}_{2} \mathrm{O}_{3}-\mathrm{IrO}_{2}$, in the $\mathrm{Nd}-\mathrm{Ir}-\mathrm{Oxygen}$ ternary system. At the lower temperatures the oxygen content of the specimens closely conform to the compositions represented by the pseudo binary $\mathrm{Nd}_{2} \mathrm{O}_{3}-\mathrm{IrO}_{2}$ system. As the temperature is increased, the compositions of the solid phases change by an apparent oxygen loss to those indicated by the $\mathrm{Nd}_{2} \mathrm{O}_{3}-\mathrm{Ir}$ join. By considering this dissociation as a type of phase transition in which the vapor phase is ignored, a simple binary representation of the phase relations in a portion of the ternary system can be given. This method of illustration has been employed by a number of investigators, notably A. Muan in his work on iron oxides $[13,14]$.

At $1020 \pm 5{ }^{\circ} \mathrm{C}$ in an air environment at atmospheric pressure, $\mathrm{IrO}_{2}$ was found to dissociate to one solid phase, Ir metal. This value favorably compares with the $1015{ }^{\circ} \mathrm{C}$ dissociation temperature at $160 \mathrm{~mm} \mathrm{Hg}$ oxygen pressure calculated from the data given by Cordfunke and Meyer in their study of the Ir-Oxygen system [5]. The dissociation of $\mathrm{IrO}_{2}$ is apparently a reversible process. For practical purposes the dissociation can be represented as a type of polymorphic transformation. 


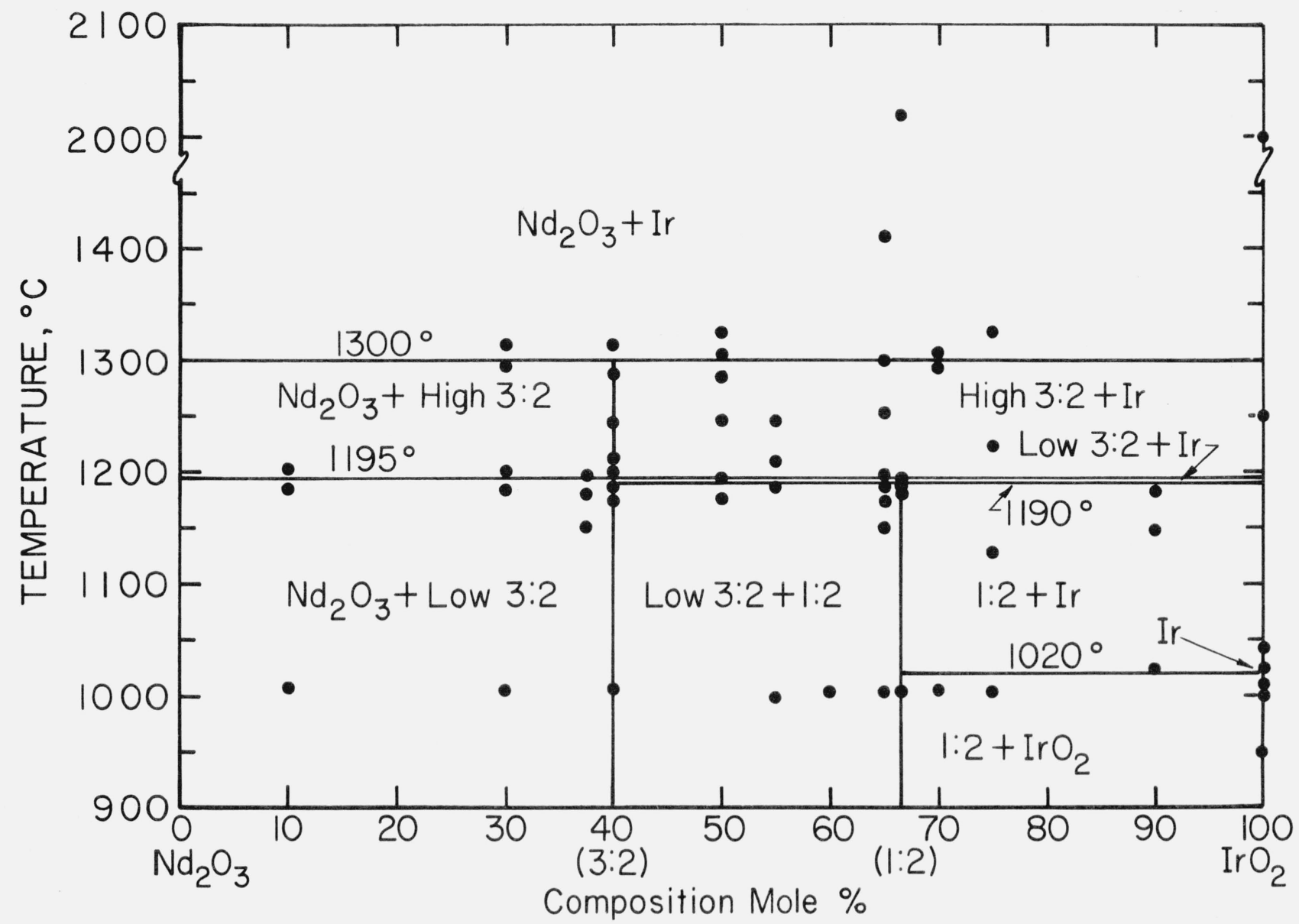

Figure 1. Composite phase equilibrium diagrams for the $\mathrm{Nd}_{2} \mathrm{O}_{3}-\mathrm{IrO}_{2}$ and $\mathrm{Nd}_{2} \mathrm{O}_{3}-\mathrm{Ir}$ systems in air.

-compositions and temperatures of experiments conducted in an air environment.

Low $3: 2-3 \mathrm{Nd}_{2} \mathrm{O}_{3} \cdot 2 \mathrm{IrO}_{2}$.

High $3: 2-3 \mathrm{Nd}_{2} \mathrm{O} \cdot 2 \mathrm{IrO}_{x} ; x$ is probably less than 2 .

$1: 2-\mathrm{Nd}_{2} \mathrm{O}_{3} \cdot 2 \mathrm{IrO}_{2}$ (pyrochlore type compound).

Ir-iridium metal.

TABLE 1. Experimental data for compositions in the $\mathrm{M}_{2} \mathrm{O}_{3}-\mathrm{IrO}_{2}$ and $\mathrm{M}_{2} \mathrm{O}_{3}-\mathrm{Ir}$ systems in air

\begin{tabular}{|c|c|c|c|c|c|}
\hline \multirow{2}{*}{ System } & \multirow{2}{*}{ Composition } & \multicolumn{2}{|c|}{ Heat treatment } & \multirow{2}{*}{$X$-ray diffraction analyses ${ }^{b}$} & \multirow{2}{*}{ Remarks } \\
\hline & & temp. ${ }^{a}$ & Time & & \\
\hline \multirow{6}{*}{$\mathrm{Nd}_{2} \mathrm{O}_{3}-\mathrm{IrO}_{2}$} & Mole \% & ${ }^{\circ} \mathrm{C}$ & $h r$ & & \\
\hline & $90: 10$ & 1009 & 19 & $\mathrm{Nd}_{2} \mathrm{O}_{3}+$ low $3 \mathrm{Nd}_{2} \mathrm{O}_{3} \cdot 2 \mathrm{IrO}_{2}$ & \\
\hline & & ${ }^{\mathrm{c}} 1185$ & 19 & $\mathrm{Nd}_{2} \mathrm{O}_{3}+$ low $3 \mathrm{Nd}_{2} \mathrm{O}_{3} \cdot 2 \mathrm{IrO}_{2}$ & \\
\hline & & 1202 & 20 & $\mathrm{Nd}_{2} \mathrm{O}_{3}+$ high $3 \mathrm{Nd}_{2} \mathrm{O}_{3} \cdot 2 \mathrm{IrO} \mathrm{O}_{2}$ & Possibly small amount of low $3: 2$ present. \\
\hline & $70: 30$ & $\begin{array}{r}1009 \\
{ }^{c} 1185 \\
{ }^{c} 1201\end{array}$ & $\begin{array}{l}19 \\
60 \\
19\end{array}$ & $\begin{array}{l}\text { Low } 3 \mathrm{Nd}_{2} \mathrm{O}_{3} \cdot 2 \mathrm{IrO}_{2}+\mathrm{Nd}_{2} \mathrm{O}_{3} \\
\text { Low } 3 \mathrm{Nd}_{2} \mathrm{O}_{3} \cdot 2 \mathrm{IrO}_{2}+\mathrm{Nd}_{2} \mathrm{O}_{3} \\
\text { Low } 3 \mathrm{Nd}_{2} \mathrm{O}_{3} \cdot 2 \mathrm{IrO}_{2}+\text { high } 3 \mathrm{Nd}_{2} \mathrm{O}_{3} \cdot 2 \mathrm{IrO}_{2} \\
\quad+\mathrm{Nd}_{2} \mathrm{O}_{3}\end{array}$ & Nonequilibrium. \\
\hline & & $\begin{array}{l}{ }^{\mathrm{c}} 1296 \\
{ }^{\mathrm{c}} 1315\end{array}$ & $\begin{array}{l}20 \\
19\end{array}$ & $\begin{array}{l}\mathrm{High} 3 \mathrm{Nd}_{2} \mathrm{O}_{3} \cdot 2 \mathrm{IrO}_{2}+\mathrm{Nd}_{2} \mathrm{O}_{3} \\
\mathrm{Nd}_{2} \mathrm{O}_{3}+\text { high } 3 \mathrm{Nd}_{2} \mathrm{O}_{3} \cdot 2 \mathrm{IrO}_{2} \\
3 \mathrm{Nd}_{2} \mathrm{O}_{3} \cdot 2 \mathrm{IrO}_{2}\end{array}$ & $\begin{array}{l}\text { Possibly small amount of low } 3: 2 \text { present. } \\
\text { Nonequilibrium: low } 3: 2 \text { probably formed } \\
\text { on cooling; Ir not detected by x rays }{ }^{\mathrm{d}} \text {; } \\
\text { high } 3: 2 \text { present only in minor amounts. }\end{array}$ \\
\hline
\end{tabular}


TABLE 1. Experimental data for compositions in the $\mathrm{M}_{2} \mathrm{O}_{3}-\mathrm{IrO}_{2}$ and $\mathrm{M}_{2} \mathrm{O}_{3}-\mathrm{Ir}$ systems in air-Continued

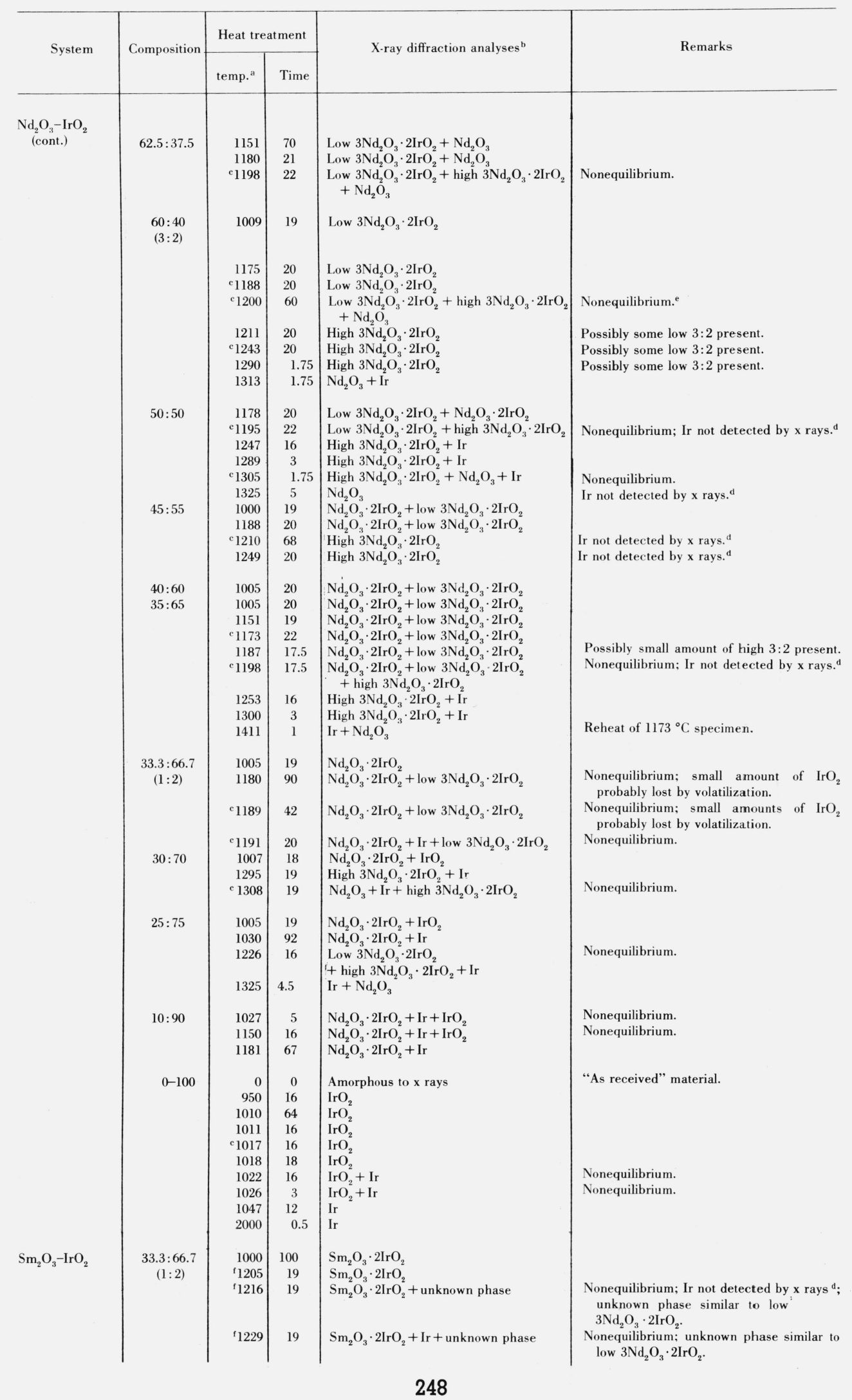


TABLE 1. Experimental data for compositions in the $\mathrm{M}_{2} \mathrm{O}_{3}-\mathrm{IrO}_{2}$ and $\mathrm{M}_{2} \mathrm{O}_{3}-\mathrm{Ir}$ systems in air-Continued

\begin{tabular}{|c|c|c|c|c|c|}
\hline \multirow{2}{*}{ System } & \multirow{2}{*}{ Composition } & \multicolumn{2}{|c|}{ Heat treatment } & \multirow{2}{*}{ X-ray diffraction analyses ${ }^{b}$} & \multirow{2}{*}{ Remarks } \\
\hline & & temp. ${ }^{a}$ & Time & & \\
\hline $\mathrm{Eu}_{2} \mathrm{O}_{3}-\mathrm{IrO}_{2}$ & $\begin{array}{l}33.3: 66.7 \\
\quad(1: 2)\end{array}$ & $\begin{array}{l}1000 \\
\text { ' } 1207 \\
\text { ' } 1215 \\
{ }^{ } 1225 \\
\text { ' } 1231 \\
\text { ' } 1346\end{array}$ & $\begin{array}{r}100 \\
64 \\
64 \\
16 \\
16 \\
19\end{array}$ & $\begin{array}{l}\mathrm{Eu}_{2} \mathrm{O}_{3} \cdot 2 \mathrm{IrO} \mathrm{O}_{2} \\
\mathrm{Eu}_{2} \mathrm{O}_{3} \cdot 2 \mathrm{IrO} \mathrm{I}_{2} \\
\mathrm{Eu}_{2} \mathrm{O}_{3} \cdot 2 \mathrm{IrO} \mathrm{O}_{2} \\
\mathrm{Eu}_{2} \mathrm{O}_{3} \cdot 2 \mathrm{IrO} \mathrm{O}_{2}+\text { unknown phase } \\
\mathrm{Eu}_{2} \mathrm{O}_{3} \cdot 2 \mathrm{IrO} \mathrm{I}_{2}+\text { unknown phase } \\
\mathrm{Eu}_{2} \mathrm{O}_{3}+\mathrm{Ir}\end{array}$ & $\begin{array}{l}\text { Nonequilibrium; Ir not detected by } x \text { ray. } \\
\text { Nonequilibrium; Ir not detected by } x \text { ray. }\end{array}$ \\
\hline $\mathrm{Gd}_{2} \mathrm{O}_{3}-\mathrm{IrO}_{2}$ & $\begin{array}{l}33.3: 66.7 \\
(1: 2)\end{array}$ & $\begin{array}{l}1000 \\
{ }^{\mathrm{f}} 1218 \\
{ }^{\mathrm{f}} 1235 \\
{ }^{\mathrm{f}} 1242\end{array}$ & $\begin{array}{r}100 \\
19 \\
19 \\
19\end{array}$ & $\begin{array}{l}\mathrm{Gd}_{2} \mathrm{O}_{3} \cdot 2 \mathrm{IrO}_{2}+\mathrm{Gd}_{2} \mathrm{O}_{3} \\
\mathrm{Gd}_{2} \mathrm{O}_{3} \cdot 2 \mathrm{IrO}_{2}+\mathrm{Gd}_{2} \mathrm{O}_{3} \\
\mathrm{Gd}_{2} \mathrm{O}_{3} \cdot 2 \mathrm{IrO}_{2}+\mathrm{Gd}_{2} \mathrm{O}_{3} \\
\mathrm{Gd}_{2} \mathrm{O}_{3} \cdot 2 \mathrm{IrO}_{2}+\mathrm{Gd}_{2} \mathrm{O}_{3}+\mathrm{Ir}\end{array}$ & $\begin{array}{l}\text { Nonequilibrium. }^{e} \\
\text { Nonequilibrium. }^{e} \\
\text { Nonequilibrium. }^{e} \\
\text { Nonequilibrium. }^{e}\end{array}$ \\
\hline $\mathrm{Dy}_{2} \mathrm{O}_{3}-\mathrm{IrO}_{2}$ & $\begin{array}{l}33.3: 66.7 \\
\quad(1: 2)\end{array}$ & $\begin{array}{l}{ }^{\mathrm{f}} 1000 \\
{ }^{\mathrm{f}} 1170 \\
{ }^{\mathrm{f}} 1200 \\
{ }^{\mathrm{f}} 1242 \\
{ }^{\mathrm{c}} 1260 \\
{ }^{\mathrm{c}} 1301 \\
{ }^{\mathrm{f}} 1351\end{array}$ & $\begin{array}{r}100 \\
92 \\
19 \\
19 \\
20 \\
4 \\
19\end{array}$ & $\begin{array}{l}\mathrm{Dy}_{2} \mathrm{O}_{3} \cdot 2 \mathrm{IrO}_{2}+\mathrm{Dy}_{2} \mathrm{O}_{3} \\
\mathrm{Dy}_{2} \mathrm{O}_{3} \cdot 2 \mathrm{IrO}_{2}+\mathrm{Dy}_{2} \mathrm{O}_{3} \\
\mathrm{Dy}_{2} \mathrm{O}_{3} \cdot 2 \mathrm{IrO}_{2}+\mathrm{Dy}_{2} \mathrm{O}_{3} \\
\mathrm{Dy}_{2} \mathrm{O}_{3} \cdot 2 \mathrm{IrO}_{2}+\mathrm{Dy}_{2} \mathrm{O}_{3} \\
\mathrm{Dy}_{2} \mathrm{O}_{3} \cdot 2 \mathrm{IrO}_{2}+\mathrm{Dy}_{2} \mathrm{O}_{3}+\text { unknown phase } \\
\mathrm{Dy}_{2} \mathrm{O}_{3} \cdot 2 \mathrm{IrO} \mathrm{Ir}_{2}+\mathrm{Dy}_{2} \mathrm{O}_{3}+\text { unknown phase } \\
\mathrm{Dy}_{2} \mathrm{O}_{3}+\mathrm{Ir}\end{array}$ & $\begin{array}{l}\text { Nonequilibrium. }{ }^{e} \\
\text { Nonequilibrium. }^{e} \\
\text { Nonequilibrium. }^{e} \\
\text { Nonequilibrium. }^{e} \\
\text { Nonequilibrium. }^{e} \\
\text { Nonequilibrium. }^{e}\end{array}$ \\
\hline $\mathrm{Ho}_{2} \mathrm{O}_{3}-\mathrm{IrO}_{2}$ & $\begin{array}{l}33.3: 66.7 \\
\quad(1: 2)\end{array}$ & $\begin{array}{l}1000 \\
{ }^{\mathrm{f}} 1218 \\
{ }^{\mathrm{f}} 1235\end{array}$ & $\begin{array}{r}100 \\
19 \\
19\end{array}$ & $\begin{array}{l}\mathrm{Ho}_{2} \mathrm{O}_{3} \cdot 2 \mathrm{IrO}_{2}+\mathrm{Ho}_{2} \mathrm{O}_{3}+\mathrm{IrO}_{2} \\
\mathrm{Ho}_{2} \mathrm{O}_{3} \cdot 2 \mathrm{IrO}_{2}+\mathrm{Ho}_{2} \mathrm{O}_{3} \\
\mathrm{Ho}_{2} \mathrm{O}_{3} \cdot 2 \mathrm{IrO}_{2}+\mathrm{Ho}_{2} \mathrm{O}_{3}+\mathrm{Ir}\end{array}$ & $\begin{array}{l}\text { Nonequilibrium. } \\
\text { Nonequilibrium. }^{e} \\
\text { Nonequilibrium. }^{e}\end{array}$ \\
\hline $\mathrm{Y}_{2} \mathrm{O}_{3}-\mathrm{IrO}_{2}$ & $\begin{array}{l}33.3: 66.7 \\
(1: 2)\end{array}$ & $\begin{array}{r}1000 \\
{ }^{\mathrm{t}} 1210 \\
{ }^{\mathrm{c}} 1216 \\
1225 \\
1351\end{array}$ & $\begin{array}{r}100 \\
19 \\
4 \\
19 \\
19\end{array}$ & $\begin{array}{l}\mathrm{Y}_{2} \mathrm{O}_{3} \cdot 2 \mathrm{IrO}_{2}+\mathrm{Y}_{2} \mathrm{O}_{3} \\
\mathrm{Y}_{2} \mathrm{O}_{3} \cdot 2 \mathrm{IrO} \mathrm{I}_{2}+\mathrm{Y}_{2} \mathrm{O}_{3} \\
\mathrm{Y}_{2} \mathrm{O}_{3} \cdot 2 \mathrm{IrO} \mathrm{I}_{2}+\mathrm{Y}_{2} \mathrm{O}_{3} \\
\mathrm{Y}_{2} \mathrm{O}_{3} \cdot 2 \mathrm{IrO} \mathrm{Ir}_{2}+\mathrm{Y}_{2} \mathrm{O}_{3}+\text { unknown phase } \\
\mathrm{Y}_{2} \mathrm{O}_{3}+\mathrm{Ir}\end{array}$ & $\begin{array}{l}\text { Nonequilibrium. } .^{e} \\
\text { Nonequilibrium. } .^{e} \\
\text { Nonequilibrium. } .^{e} \\
\text { Nonequilibrium. }{ }^{e}\end{array}$ \\
\hline $\mathrm{Er}_{2} \mathrm{O}_{3}-\mathrm{IrO}_{2}$ & $\begin{array}{l}33.3: 66.7 \\
(1: 2)\end{array}$ & $\begin{array}{r}1000 \\
{ }^{f} 1215 \\
{ }^{\mathrm{c}} 1225\end{array}$ & $\begin{array}{r}100 \\
64 \\
64\end{array}$ & $\begin{array}{l}\mathrm{Er}_{2} \mathrm{O}_{3} \cdot 2 \mathrm{IrO}_{2}+\mathrm{Er}_{2} \mathrm{O}_{3} \\
\mathrm{Er}_{2} \mathrm{O}_{3} \cdot 2 \mathrm{IrO}_{2}+\mathrm{Er}_{2} \mathrm{O}_{3} \\
\mathrm{Er}_{2} \mathrm{O}_{3} \cdot 2 \mathrm{IrO}_{2}+\mathrm{Er}_{2} \mathrm{O}_{3} \\
\mathrm{Er}_{2} \mathrm{O}_{3}+\mathrm{Ir}\end{array}$ & $\begin{array}{l}\text { Nonequilibrium. }{ }^{\mathrm{e}} \\
\text { Nonequilibrium. }^{\mathrm{e}} \\
\text { Nonequilibrium }^{\mathrm{e}}: 1: 2 \text { phase reduced in } \\
\text { amount from that of previous heat treat- } \\
\text { ment. Ir not detected by } \mathrm{x} \text { rays. }{ }^{d}\end{array}$ \\
\hline $\mathrm{Tm}_{2} \mathrm{O}_{3}-\mathrm{IrO}_{2}$ & $\begin{array}{l}33.3: 66.7 \\
(1: 2)\end{array}$ & $\begin{array}{r}1000 \\
{ }^{\mathrm{f}} 1199 \\
{ }^{\mathrm{c}} 1226\end{array}$ & $\begin{array}{r}100 \\
19 \\
19 \\
19\end{array}$ & $\begin{array}{l}\mathrm{Tm}_{2} \mathrm{O}_{3} \cdot 2 \mathrm{IrO}_{2}+\mathrm{Tm}_{2} \mathrm{O}_{3} \\
\mathrm{Tm}_{2} \mathrm{O}_{3} \cdot 2 \mathrm{IrO}_{2}+\mathrm{Tm}_{2} \mathrm{O}_{3} \\
\mathrm{Tm}_{2} \mathrm{O}_{3}+\mathrm{Tm}_{2} \mathrm{O}_{3} \cdot 2 \mathrm{IrO}_{2} \\
\\
\mathrm{Tm}_{2} \mathrm{O}_{3}+\mathrm{Ir}\end{array}$ & $\begin{array}{l}\text { Nonequilibrium. } .^{e} \\
\text { Nonequilibrium. }{ }^{e} \\
\text { Nonequilibrium }{ }^{\mathrm{e}} ; \quad 1: 2 \text { phase reduced in } \\
\text { amount from that of previous heat treat- } \\
\text { ment; Ir not detected by x rays. }{ }^{\mathrm{d}}\end{array}$ \\
\hline $\mathrm{Yb}_{2} \mathrm{O}_{3}-\mathrm{IrO}_{2}$ & $\begin{array}{l}33.3: 66.7 \\
(1: 2)\end{array}$ & $\begin{array}{r}1000 \\
{ }^{f} 1161 \\
{ }^{f} 1173 \\
{ }^{\mathrm{f}} 1199 \\
{ }^{\mathrm{f}} 1226\end{array}$ & $\begin{array}{r}100 \\
19 \\
19 \\
19 \\
19\end{array}$ & $\begin{array}{l}\mathrm{Yb}_{2} \mathrm{O}_{3} \cdot 2 \mathrm{IrO}_{2}+\mathrm{Yb}_{2} \mathrm{O}_{3} \\
\mathrm{Yb}_{2} \mathrm{O}_{3} \cdot 2 \mathrm{IrO}_{2}+\mathrm{Yb}_{2} \mathrm{O}_{3} \\
\mathrm{Yb}_{2} \mathrm{O}_{3} \cdot 2 \mathrm{IrO} \mathrm{I}_{2}+\mathrm{Yb}_{2} \mathrm{O}_{3} \\
\mathrm{Yb}_{2} \mathrm{O}_{3}+\mathrm{Ir}+\mathrm{Yb}_{2} \mathrm{O}_{3} \cdot 2 \mathrm{IrO} \\
\mathrm{Yb}_{2} \mathrm{O}_{3}+\mathrm{Ir}\end{array}$ & $\begin{array}{l}\text { Nonequilibrium. }{ }^{e} \\
\text { Nonequilibrium. }{ }^{e} \\
\text { Nonequilibrium. }{ }^{e} \\
\text { Nonequilibrium. }\end{array}$ \\
\hline $\mathrm{Lu}_{2} \mathrm{O}_{3}-\mathrm{IrO}_{2}$ & $\begin{array}{l}33.3: 66.7 \\
\quad(1: 2)\end{array}$ & $\begin{array}{l}1000 \\
{ }^{\mathrm{f}} 1161 \\
{ }^{\mathrm{f}} 1170 \\
{ }^{\mathrm{c}} 1200 \\
{ }^{\mathrm{f}} 1309\end{array}$ & $\begin{array}{r}100 \\
19 \\
19\end{array}$ & $\begin{array}{l}\mathrm{Lu}_{2} \mathrm{O}_{3} \cdot 2 \mathrm{IrO}_{2}+\mathrm{Lu}_{2} \mathrm{O}_{3} \\
\mathrm{Lu}_{2} \mathrm{O}_{3} \cdot 2 \mathrm{IrO}_{2}+\mathrm{Lu}_{2} \mathrm{O}_{3} \\
\mathrm{Lu}_{2} \mathrm{O}_{3} \cdot 2 \mathrm{IrO}_{2}+\mathrm{Lu}_{2} \mathrm{O}_{3} \\
\\
\\
\mathrm{Lu}_{2} \mathrm{O}_{3}+\mathrm{Ir}+\mathrm{Lu}_{2} \mathrm{O}_{3} \cdot 2 \mathrm{IrO}_{2} \\
\mathrm{Lu}_{2} \mathrm{O}_{3}+\mathrm{Ir}\end{array}$ & $\begin{array}{l}\text { Nonequilibrium. } \\
\text { Nonequilibrium. }^{\mathrm{e}} \\
\text { Nonequilibrium }{ }^{\mathrm{e}}: \quad 1: 2 \text { phase reduced in } \\
\text { amount from that of previous heat treat- } \\
\text { ment; Ir not detected by } \mathrm{x} \text { rays. }{ }^{\mathrm{d}} \\
\text { Nonequilibrium. }\end{array}$ \\
\hline $\mathrm{In}_{2} \mathrm{O}_{3}-\mathrm{IrO}_{2}$ & $\begin{array}{l}33.3: 66.7 \\
\quad(1: 2)\end{array}$ & $\begin{array}{l}1000 \\
1256\end{array}$ & $\begin{array}{r}100 \\
19\end{array}$ & $\begin{array}{l}\mathrm{In}_{2} \mathrm{O}_{3}+\mathrm{IrO}_{2} \\
\operatorname{In}_{2} \mathrm{O}_{3}+\mathrm{Ir}\end{array}$ & \\
\hline $\mathrm{Sc}_{2} \mathrm{O}_{3}-\mathrm{IrO}_{2}$ & $\begin{array}{l}33.3: 66.7 \\
\quad(1: 2)\end{array}$ & $\begin{array}{r}1000 \\
1256\end{array}$ & $\begin{array}{l}100 \\
19\end{array}$ & $\begin{array}{l}\mathrm{Sc}_{2} \mathrm{O}_{3}+\mathrm{IrO}_{2} \\
\mathrm{Sc}_{2} \mathrm{O}_{3}\end{array}$ & Ir not detected by $x$ rays. ${ }^{d}$ \\
\hline $\mathrm{Al}_{2} \mathrm{O}_{3}-\mathrm{IrO}_{2}$ & $\begin{array}{c}33.3: 66.7 \\
(1: 2)\end{array}$ & $\begin{array}{l}1000 \\
1256\end{array}$ & $\begin{array}{r}100 \\
19\end{array}$ & $\begin{array}{l}\mathrm{Al}_{2} \mathrm{O}_{3}+\mathrm{IrO}_{2} \\
\mathrm{Al}_{2} \mathrm{O}_{3}+\mathrm{Ir}\end{array}$ & \\
\hline $\mathrm{Nd}_{2} \mathrm{O}_{3}-\mathrm{Ir}$ & $\begin{array}{l}33.3: 66.7 \\
(1: 2)\end{array}$ & $\begin{array}{r}1000 \\
{ }^{\mathrm{c}} 1400 \\
{ }^{\mathrm{c}} 2020\end{array}$ & \begin{tabular}{r|}
12 \\
1 \\
.75
\end{tabular} & $\begin{array}{l}\mathrm{Nd}_{2} \mathrm{O}_{3} \cdot 2 \mathrm{IrO}_{2}+\mathrm{Ir}+\mathrm{IrO}_{2} \\
\mathrm{Ir}+\mathrm{Nd}_{2} \mathrm{O}_{3}+\mathrm{Nd}(\mathrm{OH})_{3} \\
\mathrm{Ir}+\mathrm{Nd}_{2} \mathrm{O}_{3}+\mathrm{Nd}(\mathrm{OH})_{3}\end{array}$ & $\begin{array}{l}\text { Nonequilibrium. } \\
\mathrm{Nd}_{2} \mathrm{O}_{3} \text { commonly hydrates at room tempera- } \\
\text { ture. } \\
\mathrm{Nd}_{2} \mathrm{O}_{3} \text { commonly hydrates at room tempera- } \\
\text { ture. }\end{array}$ \\
\hline
\end{tabular}


TABLE 1. Experimental data for compositions in the $\mathrm{M}_{2} \mathrm{O}_{3}-\mathrm{IrO}_{2}$ and $\mathrm{M}_{2} \mathrm{O}_{3}-\mathrm{Ir}$ systems in air-Continued

\begin{tabular}{|c|c|c|c|c|c|}
\hline \multirow{2}{*}{ System } & \multirow{2}{*}{ Composition } & \multicolumn{2}{|c|}{ Heat treatment } & \multirow{2}{*}{ X-ray diffraction analyses ${ }^{b}$} & \multirow{2}{*}{ Remarks } \\
\hline & & temp. ${ }^{a}$ & Time & & \\
\hline $\begin{array}{l}\mathrm{Nd}_{2} \mathrm{O}_{3}-\mathrm{Ir} \\
\text { (cont.) }\end{array}$ & $0: 100$ & $\begin{array}{r}0 \\
1000 \\
{ }^{\mathrm{c}} 1000 \\
\\
{ }^{\mathrm{c}} 1000 \\
{ }^{\mathrm{c}} 1040 \\
\\
{ }^{\mathrm{c}} 1250\end{array}$ & $\begin{array}{r}0 \\
6 \\
20 \\
\\
116 \\
18\end{array}$ & 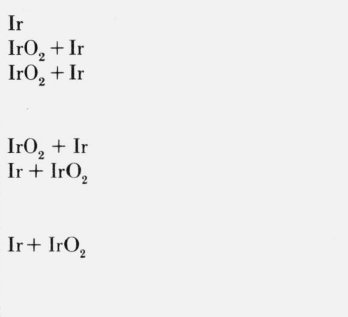 & $\begin{array}{l}\text { "As received." } \\
\text { Nonequilibrium. } \\
\text { Nonequilibrium; greater amount of } \mathrm{IrO}_{2} \\
\text { present from that of previous heat treat- } \\
\text { ment. } \\
\text { Nonequilibrium. } \\
\text { Nonequilibrium; smaller amount of } \mathrm{IrO}_{2} \\
\text { present from that of previous heat treat- } \\
\text { ment. } \\
\text { Nonequilibrium; only small amount of } \\
\mathrm{IrO} \mathrm{O}_{2} \text { present. } \mathrm{IrO}_{2} \text { probably formed on } \\
\mathrm{cooling}\end{array}$ \\
\hline $\mathrm{Sm}_{2} \mathrm{O}_{3}-\mathrm{Ir}$ & $\begin{array}{c}33.3: 66.7 \\
(1: 2)\end{array}$ & $\begin{array}{r}1000 \\
{ }^{\mathrm{c}} 1400 \\
{ }^{\mathrm{c}} 2030\end{array}$ & $\begin{array}{l}12 \\
1 \\
0.50\end{array}$ & $\begin{array}{l}\mathrm{Sm}_{2} \mathrm{O}_{3} \cdot 2 \mathrm{IrO}_{2}+\mathrm{IrO}_{2}+\mathrm{Ir}+\text { unknown } \\
\text { phase } \\
\mathrm{Sm}_{2} \mathrm{O}_{3}+\mathrm{Ir} \\
\mathrm{Sm}_{2} \mathrm{O}_{3}+\mathrm{Ir}\end{array}$ & $\begin{array}{l}\text { Nonequilibrium; unknown phase similar to } \\
\text { low } 3 \mathrm{Nd}_{2} \mathrm{O}_{3} \cdot 2 \operatorname{IrO} \mathrm{O}_{2} \text {. }\end{array}$ \\
\hline $\mathrm{Eu}_{2} \mathrm{O}_{3}-\mathrm{Ir}$ & $\begin{array}{c}33.3: 66.7 \\
(1: 2)\end{array}$ & $\begin{array}{r}1000 \\
{ }^{2} 1400 \\
{ }^{c} 2020\end{array}$ & $\begin{array}{l}12 \\
1 \\
0.50\end{array}$ & $\begin{array}{l}\mathrm{Eu}_{2} \mathrm{O}_{3} \cdot 2 \mathrm{IrO} \mathrm{O}_{2} \\
\mathrm{Eu}_{2} \mathrm{O}_{3}+\mathrm{Ir} \\
\mathrm{Eu}_{2} \mathrm{O}_{3}+\mathrm{Ir}\end{array}$ & \\
\hline $\mathrm{Gd}_{2} \mathrm{O}_{3}-\mathrm{Ir}$ & $\begin{array}{l}33.3: 66.7 \\
(1: 2)\end{array}$ & $\begin{array}{r}1000 \\
{ }^{c} 1400 \\
{ }^{c} 2015\end{array}$ & $\begin{array}{l}12 \\
1 \\
0.50\end{array}$ & $\begin{array}{l}\mathrm{Gd}_{2} \mathrm{O}_{3} \cdot 2 \mathrm{IrO}_{2}+\mathrm{Gd}_{2} \mathrm{O}_{3}+\mathrm{IrO}_{2}+\mathrm{Ir} \\
\mathrm{Gd}_{2} \mathrm{O}_{3}+\mathrm{Ir} \\
\mathrm{Gd}_{2} \mathrm{O}_{3}+\mathrm{Ir}\end{array}$ & Nonequilibrium. \\
\hline $\mathrm{Dy}_{2} \mathrm{O}_{3}-\mathrm{Ir}$ & $\begin{array}{c}33.3: 66.7 \\
(1: 2)\end{array}$ & $\begin{array}{r}1000 \\
{ }^{c} 1400 \\
{ }^{c} 2015\end{array}$ & $\begin{array}{l}12 \\
1 \\
0.50\end{array}$ & $\begin{array}{l}\mathrm{Dy}_{2} \mathrm{O}_{3} \cdot 2 \mathrm{IrO}_{2}+\mathrm{Dy}_{2} \mathrm{O}_{3}+\mathrm{IrO}_{2}+\mathrm{Ir} \\
\mathrm{Dy}_{2} \mathrm{O}_{3}+\mathrm{Ir} \\
\mathrm{Dy}_{2} \mathrm{O}_{3}+\mathrm{Ir}\end{array}$ & Nonequilibrium. \\
\hline $\mathrm{Y}_{2} \mathrm{O}_{3}-\mathrm{Ir}$ & $\begin{array}{c}33.3: 66.7 \\
(1: 2)\end{array}$ & $\begin{array}{r}1000 \\
{ }^{c} 1400 \\
2010\end{array}$ & $\begin{array}{l}12 \\
1 \\
0.50\end{array}$ & $\begin{array}{l}\mathrm{Y}_{2} \mathrm{O}_{3} \cdot 2 \mathrm{IrO}_{2}+\mathrm{Y}_{2} \mathrm{O}_{3}+\mathrm{IrO}_{2}+\mathrm{Ir} \\
\mathrm{Y}_{2} \mathrm{O}_{3}+\mathrm{Ir} \\
\mathrm{Y}_{2} \mathrm{O}_{3}+\mathrm{Ir}\end{array}$ & Nonequilibrium. \\
\hline $\mathrm{Lu}_{2} \mathrm{O}_{3}-\mathrm{Ir}$ & $\begin{array}{l}33.3: 66.7 \\
(1: 2)\end{array}$ & $\begin{array}{r}1000 \\
{ }^{1} 1400 \\
{ }^{c} 2080\end{array}$ & $\begin{array}{l}12 \\
1 \\
0.50\end{array}$ & $\begin{array}{l}\mathrm{Lu}_{2} \mathrm{O}_{3} \cdot 2 \mathrm{IrO}_{2}+\mathrm{Lu}_{2} \mathrm{O}_{3}+\mathrm{IrO}_{2}+\mathrm{Ir} \\
\mathrm{Lu}_{2} \mathrm{O}_{3}+\mathrm{Ir} \\
\mathrm{Lu}_{2} \mathrm{O}_{3}+\mathrm{Ir}\end{array}$ & Nonequilibrium. \\
\hline $\mathrm{Al}_{2} \mathrm{O}_{3}-\mathrm{Ir}$ & $\begin{array}{c}33.3: 66.7 \\
(1: 2)\end{array}$ & $\begin{array}{r}1000 \\
{ }^{\mathrm{c}} 1400 \\
2010 \\
\\
2055\end{array}$ & $\begin{array}{c}12 \\
1 \\
0.5 \\
0.5\end{array}$ & $\begin{array}{l}\mathrm{Al}_{2} \mathrm{O}_{3}+\mathrm{Ir}+\mathrm{IrO}_{2} \\
\mathrm{Al}_{2} \mathrm{O}_{3}+\mathrm{Ir} \\
\mathrm{Al}_{2} \mathrm{O}_{3}+\mathrm{Ir}+\mathrm{IrO}_{2} \\
\mathrm{Al}_{2} \mathrm{O}_{3}+\mathrm{Ir}\end{array}$ & $\begin{array}{l}\text { Nonequilibrium. } \\
\text { Nonequilibrium: } \mathrm{IrO}_{2} \text { probably formed on } \\
\text { cooling. } \\
\text { Specimen partially melted. }\end{array}$ \\
\hline
\end{tabular}

\footnotetext{
${ }^{\text {a }}$ All specimens in the $\mathrm{Nd}_{2} \mathrm{O}_{3}-\mathrm{IrO}_{2}$ system heat treated at $800{ }^{\circ} \mathrm{C}$ a minimum of $24 \mathrm{hrs}$ prior to the listed heat treatment.

${ }^{\mathrm{b}}$ The phases identified are given in order of the relative amount present at room temperature.

c Reheat of the previous specimen.

d Iridium dioxide $\left(\mathrm{IrO}_{2}\right)$ in combination with other oxides, upon
}

dissociation, forms small Ir metal grains which are generally detectable by microscopic examination. X-ray patterns of these specimens often fail to show reflections representing Ir unless there is anomalous intensity due to orientation of the grains.

e Small amount of $\mathrm{IrO}_{2}$ probably lost by volatilization.

${ }^{f}$ Reheat of $1000{ }^{\circ} \mathrm{C}$ specimen.
As $\mathrm{IrO}_{2}$ is heated to temperatures near its dissociation temperature, it becomes somewhat volatile. Cordfunke and Meyer [5] conclude that $\mathrm{IrO}_{2}$ combines with oxygen to form $\mathrm{IrO}_{3}$ which is stable only in the gaseous state. Efforts to produce solid $\mathrm{IrO}_{3}$ in the present work proved unsuccessful. The condensed vapors from heated samples of either $\mathrm{IrO}_{2}$ or Ir always were identified as $\mathrm{IrO}_{2}$. The problem of the volatility of $\mathrm{IrO}_{2}$ in the present study was minimized greatly by reacting the end members at low temperatures. The volatilization of $\mathrm{IrO}_{2}$ was sufficiently inhibited by re- action to consider the listed compositions (cation to metal ratio) of table 1 as being near correct.

Two intermediate binary compounds occur in the $\mathrm{Nd}_{2} \mathrm{O}_{3}-\mathrm{IrO}_{2}$ system. One phase, a $1: 2$ compound, is stable up to $1190{ }^{\circ} \mathrm{C}$ where it dissociates to two solid phases and a vapor phase, presumably oxygen. The composition of the $1: 2$ compound corresponds to that of an $\mathrm{A}_{2} \mathrm{~B}_{2} \mathrm{O}_{7}$ pyrochlore type phase. Table 2 gives the x-ray pattern of $\mathrm{Nd}_{2} \mathrm{O}_{3} \cdot 2 \mathrm{IrO}_{2}$ indexed on the basis of the typical face-centered cubic symmetry $(a=10.383 \AA)$ of the pyrochlore structure. 
TABLE 2. X-ray diffraction powder data for $\mathrm{Nd}_{2} \mathrm{O}_{3} \cdot 2 \mathrm{IrO}_{2}\left(\mathrm{CuK}_{\alpha}\right.$ ${\text { radiation })^{\text {a }}}$

\begin{tabular}{r|r|r}
\hline \hline$h k l^{\mathrm{b}}$ & $d^{\mathrm{c}}$ & \multicolumn{1}{c|}{$I / I_{0}{ }^{\mathrm{d}}$} \\
\hline & & \\
111 & 6.00 & 6 \\
311 & 3.140 & 4 \\
222 & 3.003 & 100 \\
400 & 2.673 & 43 \\
$511 / 333$ & 2.0035 & 3 \\
& & \\
440 & 1.8370 & 46 \\
622 & 1.5661 & 40 \\
444 & 1.4994 & 11 \\
800 & 1.2986 & 6 \\
622 & 1.1913 & 11 \\
840 & 1.1613 & 10 \\
844 & 1.0601 & 9 \\
$10,22 / 666$ & 0.9995 & 9 \\
880 & .9174 & 3 \\
$11,33 / 973$ & .8813 & 3 \\
10,62 & .8772 & 9 \\
$12,00 / 884$ & .8752 & 9 \\
12,40 & .8205 & 3 \\
\hline
\end{tabular}

${ }^{a} \mathrm{X}$-ray pattern obtained from specimen heat treated at $1005^{\circ} \mathrm{C}$ for $19 \mathrm{hrs}$ in air

b The Miller indices are those representing a fec unit cell with $a=10.383 \AA$.

Interplanar spacing.

dRelative Intensity.

The other binary phase was found to occur in the system at the $3: 2$ composition. This compound, $3 \mathrm{Nd}_{2} \mathrm{O}_{3} \cdot 2 \mathrm{IrO}_{2}$, similar in behavior to the $1: 2$ phase, also dissociates to two solid phases (and vapor) at $1300{ }^{\circ} \mathrm{C}$. At $1195{ }^{\circ} \mathrm{C}$, the $3: 2$ compound appears to undergo a polymorphic transformation. The unindexed x-ray powder patterns of both the high and low forms are given in table 3 . Because the transformation temperature of the $3: 2$ compound and the dissociation temperature of the $1: 2$ phase are approximately the same, it appears likely that an oxygen loss may have produced the apparent polymorphic phase change. Therefore, the phase designated high $3: 2$ in figure 1, may be, in reality, an oxygen deficient phase, $3 \mathrm{Nd}_{2} \mathrm{O}_{3} \cdot 2 \mathrm{IrO}$, where $x$ is less than 2 .

At temperatures above $1300{ }^{\circ} \mathrm{C}$ the system under consideration no longer can be represented by the pseudo system, $\mathrm{Nd}_{2} \mathrm{O}_{3}-\mathrm{IrO}_{2}$. Through dissociation, the system had changed to the $\mathrm{Nd}_{2} \mathrm{O}_{3}$-Ir binary. Up to temperatures of $2000{ }^{\circ} \mathrm{C}, \mathrm{Nd}_{2} \mathrm{O}_{3}$ and $\mathrm{Ir}$ do not react in the solid state. Presumably no solid state reaction would occur below melting. No definite conclusions can be made concerning the melting characteristics of the system until further experimental work has been performed.

It should be noted that figure 1 pertains only to the phase relations of the system in an air environment at atmospheric pressure. Any change in oxygen pressure would greatly change the diagram. To emphasize this, limited experiments were carried out utilizing sealed $\mathrm{Pt}$ tubes as containers. In every instance the pressure in the tube was sufficient to raise the temperatures of dissociation of mixtures of the compounds with $\mathrm{IrO}_{2} 20$ to $150^{\circ} \mathrm{C}$. For unexplained reasons pure
$\mathrm{IrO}_{2}$ could not be heated to temperatures in excess of $1000{ }^{\circ} \mathrm{C}$ without tube failure due to the high internal pressure.

TABLE 3. X-ray diffraction powder data for the "high" and "low" forms of $3 \mathrm{Nd}_{2} \mathrm{O}_{3} \cdot 2 \mathrm{IrO}_{2}\left(\mathrm{CuK}_{\alpha} \text { radiation }\right)^{\text {a }}$

\begin{tabular}{|c|c|c|c|}
\hline \multicolumn{2}{|c|}{ "Low" $3 \mathrm{Nd}_{2} \mathrm{O}_{3} \cdot 2 \mathrm{IrO}_{2}{ }^{b}$} & \multicolumn{2}{|c|}{ "High" $3 \mathrm{Nd}_{2} \mathrm{O}_{3} \cdot 2 \mathrm{IrO}_{2}$} \\
\hline$d^{\mathrm{d}}$ & $I / I_{0}{ }^{e}$ & $d^{\mathrm{d}}$ & $I / I_{0} \mathrm{e}^{2}$ \\
\hline 3.34 & 11 & 4.65 & 7 \\
\hline 3.20 & 100 & 4.06 & 32 \\
\hline 3.00 & 11 & 4.02 & 11 \\
\hline 2.85 & 16 & 3.70 & 4 \\
\hline 2.73 & 18 & 3.28 & 8 \\
\hline 2.64 & 34 & 3.12 & 7 \\
\hline 2.379 & 5 & 3.09 & 28 \\
\hline 2.199 & 5 & 2.85 & 100 \\
\hline 2.110 & 6 & 2.84 & 56 \\
\hline 2.076 & 5 & 2.72 & 4 \\
\hline 2.050 & 4 & 2.57 & 4 \\
\hline 2.013 & 5 & 2.494 & 7 \\
\hline 1.940 & 5 & 2.443 & 13 \\
\hline 1.897 & 36 & 2.424 & 13 \\
\hline 1.875 & 11 & 2.418 & 8 \\
\hline 1.840 & 4 & 2.110 & 7 \\
\hline 1.808 & 3 & 2.091 & 18 \\
\hline 1.763 & 5 & 2.030 & 13 \\
\hline 1.725 & 6 & 2.005 & 28 \\
\hline 1.633 & 13 & & \\
\hline 1.601 & 18 & & \\
\hline 1.596 & 16 & & \\
\hline 1.545 & 10 & & \\
\hline
\end{tabular}

\footnotetext{
${ }^{a}$ The $x$-ray patterns of these specimens show only broad and generally weak peaks Therefore, only the more intense, readily measurable reflections are reported.

b X-ray pattern obtained from specimen heat treated at $1009^{\circ} \mathrm{C}$ for 19 hrs in air.

${ }^{c}$ X-ray pattern obtained from specimen heat treated at $1243{ }^{\circ} \mathrm{C}$ for $20 \mathrm{hrs}$ in air. The high form may be oxygen deficient with the formula corresponding to the type $3 \mathrm{Nd}_{2} \mathrm{O}_{3} \cdot 2 \mathrm{IrO}_{x}$ where $x$ is less than two.

Interplanar Spacing.

Relative Intensity.
}

\subsection{Other $\mathrm{M}_{2} \mathrm{O}_{3}-\mathrm{IrO}_{2}$ Systems in Air}

Montmony and Bertaut [15] have previously reported the existence of pyrochlore type compounds in mixtures of rare earth sesquioxides and $\mathrm{IrO}_{2}$. In order to confirm the earlier work and to investigate these compounds more fully, $\mathrm{M}_{2} \mathrm{O}_{3}: 2 \mathrm{IrO}_{2}$ mole ratio mixtures were prepared from $\mathrm{IrO}_{2}$ and either $\mathrm{Sm}_{2} \mathrm{O}_{3}$, $\mathrm{Eu}_{2} \mathrm{O}_{3}, \mathrm{Gd}_{2} \mathrm{O}_{3}, \mathrm{Dy}_{2} \mathrm{O}_{3}, \mathrm{Ho}_{2} \mathrm{O}_{3}, \mathrm{Y}_{2} \mathrm{O}_{3}, \mathrm{Er}_{2} \mathrm{O}_{3}, \mathrm{Tm}_{2} \mathrm{O}_{3}$, $\mathrm{Yb}_{2} \mathrm{O}_{3}, \mathrm{Lu}_{2} \mathrm{O}_{3}, \mathrm{In}_{2} \mathrm{O}_{3}, \mathrm{Sc}_{2} \mathrm{O}_{3}$, and $\mathrm{Al}_{2} \mathrm{O}_{3}$.

Tables 1 and 4 summarize the results obtained for this set of experiments. All 1:2 mixtures containing the rare earth oxides and $\mathrm{Y}_{2} \mathrm{O}_{3}$ formed face-centered cubic pyrochlore type compounds. Unfortunately, some of the compositions contained, in addition to the pyrochlore type phase, small amounts of the pure rare earth oxide. In all probability an unknown quantity of $\mathrm{IrO}_{2}$ was lost by volatilization before complete combination occurred. The possibility still remains, however, that the true composition of the pyrochlore phase does not correspond to the idealized $\mathrm{Ln}_{2} \mathrm{Ir}_{2} \mathrm{O}_{7}$ formula. Evidence for "off composition" pyrochlore compounds have been reported elsewhere [16]. 


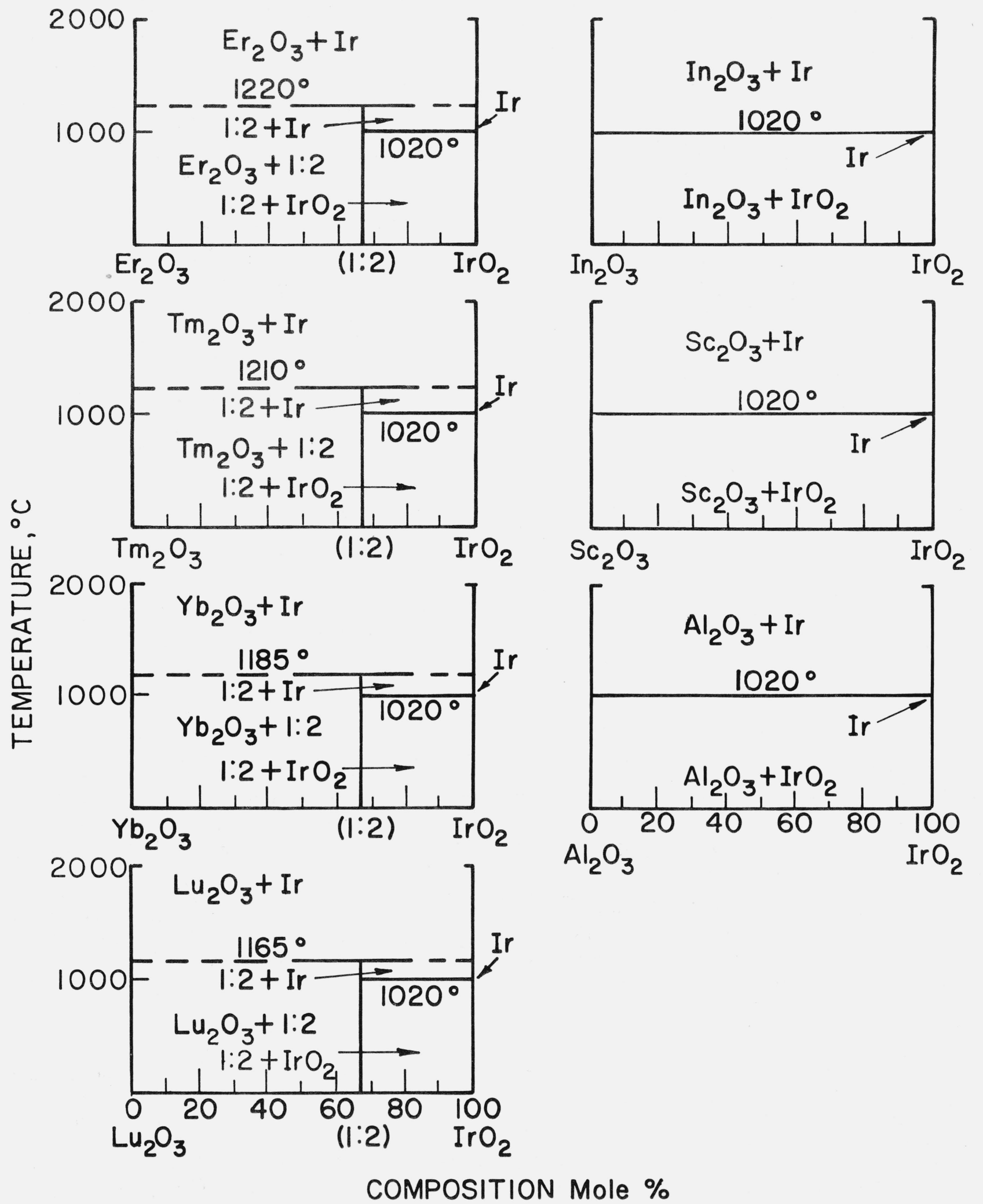

Figure 2. Predicted composite phase equilibrium diagrams for various $\mathrm{M}_{2} \mathrm{O}_{3}-\mathrm{IrO}_{2}$ and $\mathrm{M}_{2} \mathrm{O}_{3}-\mathrm{Ir}$ systems in air. 
As expected, the unit cell dimensions of the cubic pyrochlore compounds decrease in a linear manner as the size of the rare earth cation decreases. In a manner similar to $\mathrm{Nd}_{2} \operatorname{Ir}_{2} \mathrm{O}_{7}$, all the compounds dissociated at temperatures above $1020{ }^{\circ} \mathrm{C}$, the decomposition temperature of $\mathrm{IrO}_{2}$. The dissociation temperatures (table 4) when plotted as a function of the radius of the rare earth cation do not change linearly as do the unit cell dimensions. As the size of the cation is decreased, the dissociation temperature at first increases, approaching a maximum for $\mathrm{Dy}_{2} \mathrm{Ir}_{2} \mathrm{O}_{7}$ and then decreases to a minimum for $\mathrm{Lu}_{2} \mathrm{Ir}_{2} \mathrm{O}_{7}$.

Under the conditions of the experiments $\operatorname{In}_{2} \mathrm{O}_{3}$, $\mathrm{Sc}_{2} \mathrm{O}_{3}$, and $\mathrm{Al}_{2} \mathrm{O}_{3}$ did not react even partially with $\mathrm{IrO}_{2}$. X-ray patterns of these mixtures after heat treatment at $1000{ }^{\circ} \mathrm{C}$ show only reflections representing $\mathrm{IrO}_{2}$ and the admixture oxide. Additional heat treatment at $1256{ }^{\circ} \mathrm{C}$ failed to cause combination and only succeeded in dissociating the $\mathrm{IrO}_{2}$. The resultant mixture contained the refractory oxide and Ir metal.

It is evident that the $\mathrm{Nd}_{2} \mathrm{O}_{3}-\mathrm{IrO}_{2}$ system is representative in a general way of the other $\mathrm{M}_{2} \mathrm{O}_{3}-\mathrm{IrO}_{2}$ systems. Using it as a guide, figure 2 gives a number of the subsolidus phase diagrams for various systems as predicted from the data tabulated in table 1 .

The $\mathrm{In}_{2} \mathrm{O}_{3}-\mathrm{IrO}_{2}, \mathrm{Sc}_{2} \mathrm{O}_{3}-\mathrm{IrO}_{2}$, and $\mathrm{Al}_{2} \mathrm{O}_{3}-\mathrm{IrO}_{2}$ systems are rather simple and straightforward inasmuch as there is no detectable reaction between end members in the solid state. These diagrams indicate only the dissociation of $\mathrm{IrO}_{2}$, the point at which the system reverts to the true $\mathrm{M}_{2} \mathrm{O}_{3}-\mathrm{Ir}$ system.

The diagrams for the $\mathrm{Er}_{2} \mathrm{O}_{3}-\mathrm{IrO}_{2}, \mathrm{Tm}_{2} \mathrm{O}_{3}-\mathrm{IrO}_{2}$, $\mathrm{Yb}_{2} \mathrm{O}_{3}-\mathrm{IrO}_{2}$, and $\mathrm{Lu}_{2} \mathrm{O}_{3}-\mathrm{IrO}_{2}$ systems in air are slightly more complicated, each indicating the occurrence of a $1: 2$ compound. Studies of $1: 2$ compounds show that they dissociate to the solid phases $\mathrm{M}_{2} \mathrm{O}_{3}$ and Ir. If other phases occurred in the systems, they necessarily would have to dissociate at lower temperatures than the $1: 2$ compound. If other compounds existed which were stable at temperatures greater than the $1: 2$ dissociation temperatures, they would appear as a decomposition product of the pyrochlore type phase. As stated previously, this was not the case. On the basis of the more complete $\mathrm{Nd}_{2} \mathrm{O}_{3}-\mathrm{IrO}_{2}$ study, the existence of phases other than the $1: 2$ is not considered likely for these systems.

Additional experimentation is needed before the $\mathrm{Sm}_{2} \mathrm{O}_{3}-\mathrm{IrO}_{2}, \mathrm{Eu}_{2} \mathrm{O}_{3}-\mathrm{IrO}_{2}, \mathrm{Gd}_{2} \mathrm{O}_{3}-\mathrm{IrO}_{2}, \mathrm{Dy}_{2} \mathrm{O}_{3}-\mathrm{IrO}_{2}$, $\mathrm{Ho}_{2} \mathrm{O}_{3}-\mathrm{IrO}_{2}$, and $\mathrm{Y}_{2} \mathrm{O}_{3}-\mathrm{IrO}_{2}$ systems can be drawn. Each of the 1:2 compounds occurring in these systems dissociates to a mixture of solid phases, generally consisting of Ir and an unidentified phase.

\section{3. $\mathrm{M}_{2} \mathrm{O}_{3}-\mathrm{Ir}$ Reactions}

In order to establish whether or not the results found for the $\mathrm{M}_{2} \mathrm{O}_{3}-\mathrm{IrO}_{2}$ studies were indicative of $\mathrm{Ir}$ in ' combination with various oxides, a limited number of experiments were performed involving oxide-Ir mixtures. Table 1 gives the data obtained for $1: 2$ mole ratio (oxide:metal) mixtures heat treated in an air environment. It is apparent that Ir oxidizes at least partially to $\mathrm{IrO}_{2}$, which, in turn reacts with the admixture oxide to form the same pyrochlore type compounds listed in table 4 . At higher temperatures the pyrochlore type compounds dissociated as expected and further reaction between end members did not take place.

TABLE 4. $\quad \mathrm{Ln}_{2} \mathrm{O}_{3} \cdot 2 \mathrm{IrO}_{2}$ pyrochlore-type compounds

\begin{tabular}{|c|c|c|c|c|}
\hline \multirow{2}{*}{ Compound } & \multirow{2}{*}{$\begin{array}{c}\text { Radius of } \\
\mathrm{Ln}^{+3} \text { a }\end{array}$} & \multirow{2}{*}{$\begin{array}{l}\text { Dissocia- } \\
\text { tion tem- } \\
\text { perature }^{b}\end{array}$} & \multicolumn{2}{|r|}{ X-ray data } \\
\hline & & & Symmetry & Unit cell dimension \\
\hline & $\AA$ & ${ }^{\circ} \mathrm{C}$ & & $\AA$ \\
\hline $\mathrm{Nd}_{2} \mathrm{O}_{3} \cdot 2 \mathrm{IrO}_{2}$ & 1.04 & 1190 & cubic & 10.383 \\
\hline $\mathrm{Sm}_{2} \mathrm{O}_{3} \cdot 2 \mathrm{IrO}_{2}$ & 1.00 & 1210 & cubic & 10.313 \\
\hline $\mathrm{Eu}_{2} \mathrm{O}_{3} \cdot 2 \mathrm{IrO}_{2}$ & 0.98 & 1220 & cubic & 10.293 \\
\hline $\mathrm{Gd}_{2} \mathrm{O}_{3} \cdot 2 \mathrm{IrO}_{2}$ & .97 & 1240 & cubic & 10.265 \\
\hline $\mathrm{Dy}_{2} \mathrm{O}_{3}^{3} \cdot 2 \mathrm{IrO}_{2}^{2}$ & .92 & 1250 & cubic & 10.207 \\
\hline $\mathrm{Ho}_{2} \mathrm{O}_{3} \cdot 2 \mathrm{IrO}_{2}$ & .91 & 1225 & cubic & 10.180 \\
\hline $\mathrm{Y}_{2} \mathrm{O}_{3} \cdot 2 \mathrm{IrO}_{2}$ & $\approx .91$ & 1220 & cubic & 10.177 \\
\hline $\mathrm{Er}_{2} \mathrm{O}_{3} \cdot 2 \mathrm{IrO}{ }_{2}$ & .89 & 1220 & cubic & 10.163 \\
\hline $\mathrm{Tm}_{2} \mathrm{O}_{3}^{3} \cdot 2 \mathrm{Ir} \mathrm{O}_{2}^{2}$ & .87 & 1210 & cubic & 10.134 \\
\hline $\mathrm{Yb}_{2} \mathrm{O}_{3}^{3} \cdot 2 \mathrm{IrO}_{2}^{2}$ & .86 & 1185 & cubic & 10.115 \\
\hline $\mathrm{Lu}_{2} \mathrm{O}_{3} \cdot 2 \mathrm{IrO}$ & .85 & 1165 & cubic & 10.096 \\
\hline
\end{tabular}

a All radii of the rare earth cations taken from Arhens [17] with the exception of $\mathrm{Y}+3$ which was estimated by Roth and Schneider [4]

${ }^{\mathrm{b}}$ Dissociation temperatures are applicable only to those specimens heated in air.

Unfortunately, Ir either alone or in combination with an oxide, never completely oxidized to $\mathrm{IrO}_{2}$. As indicated by microscopic examination, $\mathrm{IrO}_{2}$, as it forms, appears to coat the Ir grains and thus tends to inhibit additional oxidation. At temperatures just below the $\mathrm{IrO}_{2}$ dissociation temperature, $\mathrm{IrO}_{2}$ is somewhat volatile. As the metal forms $\mathrm{IrO}_{2}$, the oxide is lost by vaporization and a steady state is reached where the metal:oxide $\left(\mathrm{Ir}: \mathrm{IrO}_{2}\right)$ ratio of the remaining material appears to remain approximately constant. In essence, true equilibrium at low temperatures could not be obtained in the $\mathrm{M}_{2} \mathrm{O}_{3}: 2 \mathrm{Ir}$ mixtures because of the inherent difficulty in achieving complete oxidation of Ir. Except for the presence of Ir at low temperatures, the data for the $\mathrm{M}_{2} \mathrm{O}_{3}: 2 \mathrm{Ir}$ compositions completely substantiated the $\mathrm{M}_{2} \mathrm{O}_{3}-\mathrm{IrO}_{2}$ studies. Also, these data, in effect, establish the reversibility of the various transformations (dissociation) discussed earlier.

In all the aforementioned experiments, every effort was made through thorough mixing and prolonged heat treatment to react Ir with the various oxides. Even with these precautions, Ir while in the metallic state appears to be unreactive with the oxides studied and thus seems quite acceptable as a container material for high temperature application in an air environment. 
The fact that Ir oxidizes at low temperatures does not appreciably detract from its use as a container since all phases containing $\mathrm{IrO}_{2}$ subsequently dissociate.

\subsection{Summary}

Selected mixtures in systems involving $\mathrm{Ir}$ or $\mathrm{IrO}_{2}$ and various sesquioxides were studied by $\mathrm{x}$-ray diffraction techniques after heat treatment in an air environment. Iridium, in air, oxidizes almost completely to $\mathrm{IrO}_{2}$ at low temperatures. The dissociation temperature of $\mathrm{IrO}_{2}$ in air at atmospheric pressure was established at $1020 \pm 5{ }^{\circ} \mathrm{C}$. Iridium in combination with the sesquioxides also forms $\mathrm{IrO}_{2}$ which in turn reacts with a number of the oxides to form binary compounds. The pseudo binary system $\mathrm{Nd}_{2} \mathrm{O}_{3}-\mathrm{IrO}_{2}$ exemplified the typical type of reaction and was studied in detail. Two phases, a compound believed to be $3 \mathrm{Nd}_{2} \mathrm{O}_{3} \cdot 2 \mathrm{IrO}_{2}$ having unknown symmetry and a cubic pyrochlore type compound $\mathrm{Nd}_{2} \mathrm{O}_{3} \cdot 2 \mathrm{IrO}_{2}$, with $a=10.383 \AA$, occur in the system. The $3: 2$ and $1: 2$ compounds dissociate at 1300 and $1190{ }^{\circ} \mathrm{C}$ respectively. Above $1300{ }^{\circ} \mathrm{C}$ the system corresponds to the $\mathrm{Nd}_{2} \mathrm{O}_{3}-\mathrm{Ir}$ join in the $\mathrm{Nd}-\mathrm{Ir}-\mathrm{O}$ ternary system. No further reaction appears to take place between $\mathrm{Nd}_{2} \mathrm{O}_{3}$ and $\mathrm{Ir}$ up to $2000{ }^{\circ} \mathrm{C}$.

Pyrochlore type compounds also were found to occur at the $1: 2$ mixture of either $\mathrm{Sm}_{2} \mathrm{O}_{3}, \mathrm{Eu}_{2} \mathrm{O}_{3}$, $\mathrm{Gd}_{2} \mathrm{O}_{3}, \mathrm{Ho}_{2} \mathrm{O}_{3}, \mathrm{Y}_{2} \mathrm{O}_{3}, \mathrm{Dy}_{2} \mathrm{O}_{3}, \mathrm{Er}_{2} \mathrm{O}_{3}, \mathrm{Tm}_{2} \mathrm{O}_{3}, \mathrm{Yb}_{2} \mathrm{O}_{3}$, or $\mathrm{Lu}_{2} \mathrm{O}_{3}$ with $\mathrm{IrO}_{2}$. Each of these compounds also dissociated upon heating at temperatures above the dissociation temperature of $\mathrm{IrO}_{2}$. Mixtures of either $\mathrm{In}_{2} \mathrm{O}_{3}, \mathrm{Sc}_{2} \mathrm{O}_{3}$, or $\mathrm{Al}_{2} \mathrm{O}_{3}$ with $\mathrm{IrO}_{2}$ did not react at temperatures up to $2000{ }^{\circ} \mathrm{C}$. The phase diagrams for a number of the $\mathrm{M}_{2} \mathrm{O}_{3}-\mathrm{IrO}_{2}$ systems have been predicted.

\section{References}

[1] S. J. Schneider, Mono. 68, NBS, 31 p. (1963).

[2] H. E. Swanson, R. K. Fuyat and G. M. Ugrinic, NBS Cir. 539, 4, 9-10 (1963).

[3] H. F. Stimson, J. Res. NBS 65A (Phys. and Chem.) No. 3, 139-145 (1960).

[4] H. E. Swanson, M. C. Morris and E. H. Evans, NBS Mono. 25, Sec. 4 (1965) to be published.

[5] E. H. P. Cordfunke and G. Meyer, Rec. Trav. Chim. 81, 495-504 (1962).

[6] R. S. Roth and S. J. Schneider, J. Res. NBS 64A (Phys. and Chem.) No. 4, 309-318 (1960).

[7] M. W. Shafer and R. Roy, J. Am. Ceram. Soc. 42, 563-570 (1959).

[8] H. E. Swanson, R. K. Fuyat and G. M. Ugrinic, NBS Cir. 539, 4, 26 (1963).

[9] W. A. Lambertson and F. H. Gunzel, Jr., A.N.L. U.S. AEC Publ. AECD-3465, 1-4 (1952).

[10] S. J. Schneider and R. S. Roth, J. Res. NBS 65A (Phys. and Chem.) No. 4, 317-332 (1960).

[11] S. J. Schneider, J. Res. NBS 65A (Phys. and Chem.) No. 5, 429-434 (1961).

[12] S. J. Schneider and J. L. Waring, J. Res. NBS 67A (Phys. and Chem.) No. 1, 19-25 (1963).

[13] A. Muan, Am. J. Sci. 256 171-207 (1958)

[14] A. Muan and C. L. Gee, J. Am. Ceram. Soc. 39 (6) 207-214 (1956).

[15] M. Montmony and F. Bertaut, Compt. Rend. $2524171-4173$ (1961).

[16] R. S. Roth, J. Res. NBS 62A (Phys. and Chem.) No. 1, 27-38 (1959).

[17] L. H. Ahrens, Geochim. et Cosmochim. Acta 2 155-169 (1952).

(Paper 69A3-343) 